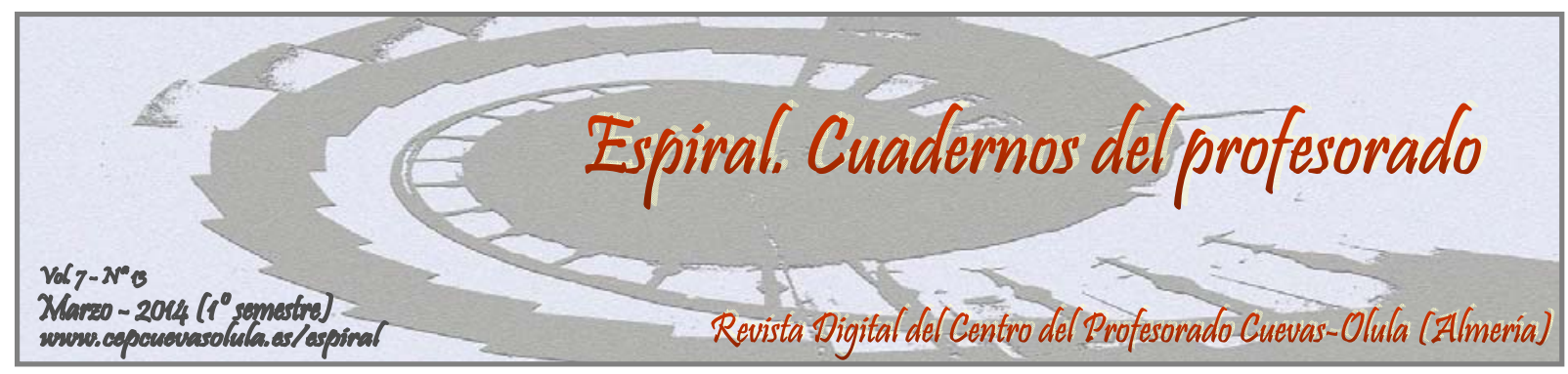

\title{
FACTORES ESTRATÉGICOS Y MOTIVACIONALES COMO PREDICTORES DEL ÉXITO EN RESOLUCIÓN DE PROBLEMAS EN MAESTRAS Y MAESTROS EN FORMACIÓN
}

\section{STRATEGIC AND MOTIVATIONAL FACTORS AS PREDICTORS OF PRE- SERVICE PRIMARY TEACHERS' SUCCESS IN PROBLEM SOLVING}

\section{Alexandre Caballer Alonso y Joan Josep Solaz Portolés}

\author{
Departamento de Didáctica de las Ciencias Experimentales y Sociales, Facultad de Magisterio, \\ Universidad de Valencia, España
}

\begin{abstract}
RESUMEN: En este trabajo se presenta una investigación descriptiva que muestra las relaciones entre cinco factores motivacionales y estratégicos (planificación, estrategias cognitivas, autocontrol, esfuerzo y autoeficacia) y el rendimiento en la resolución de problemas. Se administró a 66 maestras y maestros de primaria en formación un cuadernillo con dos problemas (problema 1 y problema 2) y el test de O'Neil y Schacter. Los resultados de los análisis de regresión múltiple ponen de manifiesto que: a) planificación, esfuerzo y autoeficacia son predictores significativos de las puntuaciones de las puntuaciones del problema de estructura simple (problema 1); b) esfuerzo, autoeficacia y estrategias cognitivas son predictores significativos de las puntuaciones del problema de estructura complicada (problema 2); c) Los coeficientes de regresión de esfuerzo y autoeficacia son negativos en el problema 1; y d) El coeficiente de regresión de esfuerzo es negativo en el problema 2. Todo ello puede permitir concluir que el éxito en la resolución de problemas del profesorado de primaria en formación depende de dos factores estratégicos, las estrategias cognitivas y la planificación (estrategia metacognitiva), y de dos factores motivacionales, el esfuerzo y la autoeficacia.
\end{abstract}

Palabras clave: resolución de problemas, maestros de primaria en formación, motivación, estrategias.

ABSTRACT: In this paper we present a descriptive research that shows the relationships between five motivational and strategic factors (planning, cognitive strategy, self-checking, effort and selfefficacy) and problem solving success. A booklet containing two word problems (problem 1 and problem 2) that differ from each other in structure (simple or complex) and the O'Neil and Schacter's test were administered to 66 third-year pre-service primary teachers. The results of the multiple regression analysis showed that: a) planning, effort and self-efficacy are significant predictors of structurally simple problem scores (problem 1); b) effort, self-efficacy and cognitive strategy are significant predictors of structurally complex problem scores (problem 2); c) The effort and self-efficacy's regression coefficients are negatives in the problem 1; and d) The effort's regression coefficient is negative in the problem 2. It can be concluded from this that pre-service primary teachers' success in problem solving depends on two strategic factors, cognitive strategy and planning (metacognitive strategy), and two motivational factors, effort and self-efficacy

Key words: problem solving, pre-service primary teachers, motivation, strategies. 
Caballer Alonso, A. y Solaz Portolés, J. J. (2014). Factores estratégicos y motivacionales como predictores del éxito en resolución de problemas en maestras y maestros en formación. Espiral. Cuadernos del Profesorado, 7(13), 20-29. Disponible en: http://www.cepcuevasolula.es/espiral.

Fecha de recepción: 03/11/2013

Fecha de aceptación: 27/02/2014
Enviar correspondencia a: joan.solaz@uv.es

\section{1.- INTRODUCCIÓN}

Desde el punto de vista de la psicología cognitiva el aprendizaje depende de la capacidad de procesar la información de una base de conocimientos apoyada sobre habilidades cognitivas y metacognitivas. Las estrategias cognitivas y metacognitivas están íntimamente relacionadas en tanto que ambas están implicadas en la cognición humana; sin embargo, son conceptualmente distintas. Así, de acuerdo con Correa, Castro y Lira (2004), las estrategias cognitivas son modalidades de trabajo intelectual que permiten adquirir, codificar y recuperar información; y las estrategias metacognitivas permiten hacer consciente y autorregular los procesos cognitivos que ejecutamos, tomar decisiones más efectivas y lograr un aprendizaje en profundidad. Flavell (1996), por su parte, afirma que la función principal de una estrategia cognitiva es ayudar a alcanzar la meta de cualquier empresa cognitiva, y una estrategia metacognitiva tiene como función informar sobre la empresa o el propio progreso. Las primeras ayudan a hacer un progreso cognitivo y las segundas a controlarlo.

Se ha constatado que los y las estudiantes que hacen uso de estrategias de estudio en las que se intenta dar estructura y organización a la nueva información, además de relacionarla con lo que ya saben, generan un mejor conocimiento proposicional o conceptual y resuelven mejor los problemas (Hegarty-Hazel y Prosser, 1991). Por otro lado, en un estudio sobre la comprensión lectora y resolución de problemas (Ferguson-Hessler y de Jong, 1990) se concluye que el alumnado que mejor resuelve los problemas es aquel que procesa la información textual con más profundidad, esto es, el que es capaz de integrar los diversos contenidos y obtener un significado global del texto. También resuelven mejor los problemas los y las estudiantes que tienen la habilidad de discriminar la información relevante de la menos importante (Johnstone, Hogg y Ziane, 1993).

Por su parte, Bielaczyc, Pirolli y Brown (1995) llevaron a cabo una investigación en la cual se enseñaba al alumnado estrategias cognitivas y metacognitivas, y como resultado de este proceso, mejoraban significativamente en las tareas de aprendizaje. Neto y Valente (1997) hallaron que el adiestramiento en el uso de estrategias metacognitivas en la resolución de problemas de física de un grupo de estudiantes conducía a mejores resultados tanto en problemas cualitativos como cuantitativos. Teong (2003) también puso a prueba el efecto del entrenamiento metacognitivo sobre la resolución de problemas. El estudiantado del grupo experimental, entrenado para llevar a cabo decisiones de carácter metacognitivo y exponerlas, superaron al grupo de control en la puntuaciones de la prueba de resolución de problemas. No obstante, es necesario tener en cuenta que las estrategias dependen de la voluntad y la autorregulación del estudiante (Weinstein y Meyer,1994), y que solamente pueden ser adquiridas a través del aprendizaje individual sobre uno mismo y nuestras destrezas, con la correspondiente carga de (auto)responsabilidad que esto comporta (Conford, 2002).

Según Wolters y Rosenthal (2000) la motivación se define como el conjunto de recursos que animan a una persona a implicarse en una tarea o a alcanzar un objetivo. En el ámbito académico, la motivación es la responsable de que los estudiantes y las estudiantes trabajen para llegar a determinados resultados. Por otro lado, estos autores sostienen que la motivación está generada por:

- Creencias sobre la autoeficacia, que son nuestras ideas sobre nuestra habilidad para planificar y ejecutar acciones que conduzcan hacia el logro de un determinado objetivo.

- Creencias sobre el valor de la tarea o evaluación que hacemos sobre el rendimiento que obtendremos del trabajo a realizar.

- Orientación hacia objetivos, que se refiere a la percepción de los motivos por los que vale 
la pena implicarnos en una tarea específica. Se puede distinguir una orientación hacia objetivos íntrínseca y otra extrínseca. La primera se da cuando un estudiante percibe que la participación en una actividad se produce por un reto personal, por curiosidad o por objetivos de aprendizaje concretos. En la segunda la participación viene guiada por motivos relacionados con la valoración y reconocimiento que otros darán al trabajo llevado a cabo, así como la entrada en competencia con otros.

Huitt (2001) categoriza las fuentes de motivación en extrínsecas e intrínsecas. Las intrínsecas las divide en función de su origen en: biológicas, mentales (cognitivas, afectivas y conativas) y transpersonales o espirituales. Las extrínsecas tienen su origen en el condicionamiento operante y el reconocimiento social. Schraw, Crippen y Hartley (2006) indican que la motivación comprendería las creencias y las actitudes que afectan al uso y desarrollo de las habilidades cognitivas y metacognitivas. Se ha comprobado que las personas con elevada necesidad de cognición tienen una motivación intrínseca en la realización de tareas complejas (Cacciopo y Petty, 1982).

Es de señalar que, sin embargo, las herramientas mas frecuentemente utilizadas en el diseño instruccional pasan por alto la motivación (Brooks y Shell, 2006) y los científicos de la cognición han ignorado durante mucho tiempo la relación entre los procesos cognitivos y la motivación (Wieth y Burns, 1999). De hecho, fue en la última década del siglo pasado cuando los investigadores y las investigadoras dirigieron su atención a la relación entre motivación y cognición, y trabajos como el de Locke y Latham (1990) muestran que parece existir una interacción entre ambas.

Diversos estudios han constatado la influencia de factores motivacionales tanto en el éxito académico (Brooks y Shell, 2006; Coutinho, 2007), como en la resolución de problemas (Wieth y Burns, 1999; Coutinho, Wiemer-Hastings, Skowronski y Britt, 2005; Marcou y Philippou, 2005; Beal y Stevens, 2007). Por otra parte, en el trabajo de Schraw, Brooks y Crippen (2005) se destacan las correlaciones existentes entre motivación, metacognición y aprendizaje. En esta línea, se ha encontrado una correlación significativa entre la necesidad de cognición (una componente motivacional) y la metacognición (Coutinho, 2006); y entre la necesidad de cognición y el éxito en la resolución de problemas (Coutinho et al., 2005). El trabajo de Marcou y Philippou (2005) se obtiene una relación de elevado nivel de confianza entre creencias motivacionales y aprendizaje autorregulado (metacognición), así como una relación estadísticamente significativa entre autoeficacia y orientación intrínseca hacia objetivos (ambas componentes motivacionales) y desempeño en la resolución de problemas.

Song y Grabowski (2009) han puesto en evidencia la posibilidad de promover la motivación intrínseca en la resolución de problemas mediante contextos de orientación hacia el aprendizaje y el trabajo en grupos de estudiantes. El contexto de orientación hacia el aprendizaje utilizado por estas autoras en su investigación se sitúa en un diseño instruccional que incluye "mensajes" donde se informa a los estudiantes de la importancia de abordar determinadas actividades "desafiantes" y del valor de lo que están aprendiendo per se. Una revisión exhaustiva de variables cognitivas, metacognitivas y motivacionales que influyen en la resolución de problemas puede encontrarse en el trabajo de Solaz-Portolés, Sanjosé y Gangoso (2013).

Los trabajos en la literatura en los que se investiga cómo los maestros y las maestras (en ejercicio y en formación) resuelven problemas están centrados en el área de las matemáticas. El conocimiento matemático de este colectivo de docentes suele decirse que es insuficiente (Simon, 1993). Brown, McNamara, Hanley y Jones (1999) apuntan que la comprensión de las matemáticas y

su enseñanza en el profesorado de primaria están muy influidas por sus experiencias académicas previas, que generalmente han sido percibidas como difíciles y amenazantes.

Taplin (1998), a partir de la constatación de las dificultades del profesorado de primaria en formación en la resolución de problemas, realizó un estudio en el que categorizó los errores que suelen cometer este estudiantado en cuatro tipos:

- De traducción de la información del enunciado del problema al lenguaje matemático.

- De interpretación del enunciado 
- De insuficiencia de conocimiento conceptual matemático

- De operaciones aritméticas.

Esta autora pone de relieve, además, que el profesorado de primaria en formación hace mejor los problemas de pensamiento icónico, donde toda la información necesaria para resolverlo está en el enunciado y solamente se necesita un paso para llegar a la solución. En cambio, resuelve peor los problemas que requieren pensamiento simbólico y relacional, donde se usan conceptos previos (que no figuran en el enunciado del problema), y en los que se hace necesario el uso de varios pasos para llegar a la solución. Por su parte, Valverde y Castro (2009) confirman el predominio de un razonamiento preproporcional en la resolución de problemas de proporcionalidad directa llevada a cabo por dicho profesorado. Así, observan una separación entre la representación simbólica y el significado de la fracción como razón.

Teniendo presentes la importancia de la resolución de problemas en el contexto educativo y en la formación de futuros maestros y maestras de primaria y las deficiencias observadas en dicho colectivo en este punto, resulta de especial interés analizar algunos factores que pueden influir en los procesos que se ejecutan en esta tarea. El objetivo del presente trabajo, centrado en el profesorado de primaria en formación, es investigar qué factores relacionados con la motivación y las estrategias predicen significativamente el rendimiento en la resolución de problemas.

\section{2.- METODOLOGÍA}

\section{Diseño experimental}

El diseño experimental utilizado es transaccional o transversal descriptivo, porque las medidas se toman en una sola ocasión. Las variables independientes serán las puntuaciones obtenidas en los cinco factores de un test de motivación y estrategias en resolución de problemas: esfuerzo, autoeficacia, estrategias cognitivas, planificación y autocontrol. La variable dependiente será la puntuación obtenida en resolución de problemas.

\section{Participantes}

Intervinieron en la investigación 66 estudiantes de ambos sexos (44 mujeres y 22 hombres) de edades comprendidas entre los 20 y los 36 años, estudiantes del tercer curso del Grado de Maestro en Educación Primaria, y pertenecientes a dos grupos de la Facultat de Magisteri de la Universitat de València.

Este estudiantado no parece tener, a priori, características especiales que lo diferencie de otros grupos del tercer curso. No obstante, hemos de indicar que no se realizó muestreo aleatorio alguno, ya que se trató de una muestra de conveniencia. Por ello, los resultados no pueden ser extrapolados a toda la población estudiantil de tercero del Grado, esto es, no hay garantías de validez externa.

\section{Materiales}

Se elaboró un cuadernillo con dos problemas (problema 1 y problema 2) que difieren en su estructura (Anexo 1). Ambos problemas tienen una estructura de proporcionalidad directa entre las variables y se resuelven mediante reglas de tres simples directas. La diferencia entre el primer y segundo problema radica en el número de reglas de tres simple directas que se han de llevar a cabo. En el caso del primer problema, solamente se ha de hacer una; en cambio, en el segundo hay que hacer como mínimo dos. Por consiguiente, el primer problema podemos calificarlo de "estructura sencilla” y el segundo de "estructura complicada".

Para medir la motivación y las estrategias del estudiantado en resolución de problemas se utilizó el test O'Neil y Schacter (1997), en concreto la traducción al castellano del test que validaron los autores. Consta de 40 ítems con los que se pretende evaluar cinco factores que pueden incidir sobre la resolución de problemas: esfuerzo, autoeficacia (ambos factores relacionados con la motivación), estrategias cognitivas, planificación y autocontrol (estos dos últimos factores constituyen estrategias metacognitivas). Utiliza una escala tipo Likert de cuatro niveles de respuesta, desde "casi nunca", que 
tiene valor 1, hasta “casi siempre”, que tiene valor 4 (Anexo 2). Los ocho ítems de cada uno de los 5 factores se encuentran repartidos aleatoriamente entre los cuarenta.

\section{Procedimiento}

Tanto el test de estrategias y motivación como el cuadernillo de los problemas se administraron en una sesión de clase normal. Se pasó en primer lugar el test de estrategias y motivación, y a continuación el cuadernillo de problemas.

Para la calificación de los problemas se confeccionó un protocolo de corrección elaborado por los autores de este trabajo y revisado por dos profesores universitarios. La calificación máxima para cada problema es de 10 puntos. La evaluación de los problemas se realizó entre un autor de este trabajo y un profesor universitario, llegándose a una coincidencia en la puntuación en el 95\% de los casos, y resolviéndose las discrepancias de mutuo acuerdo.

En el caso del test de estrategias y motivación, se obtuvo para cada estudiante la puntuación en cada uno de los 5 factores considerados (esfuerzo, autoeficacia, estrategias cognitivas, planificación y autocontrol) a partir de la media aritmética de los 8 ítems relacionados con cada factor.

\section{3.- RESULTADOS}

En la Figura 1 se representan gráficamente las puntuaciones medias en cada unos de los factores del test O'Neil y Schacter. En la Figura 2 se representan gráficamente las puntuaciones medias en los problemas 1 y 2 .

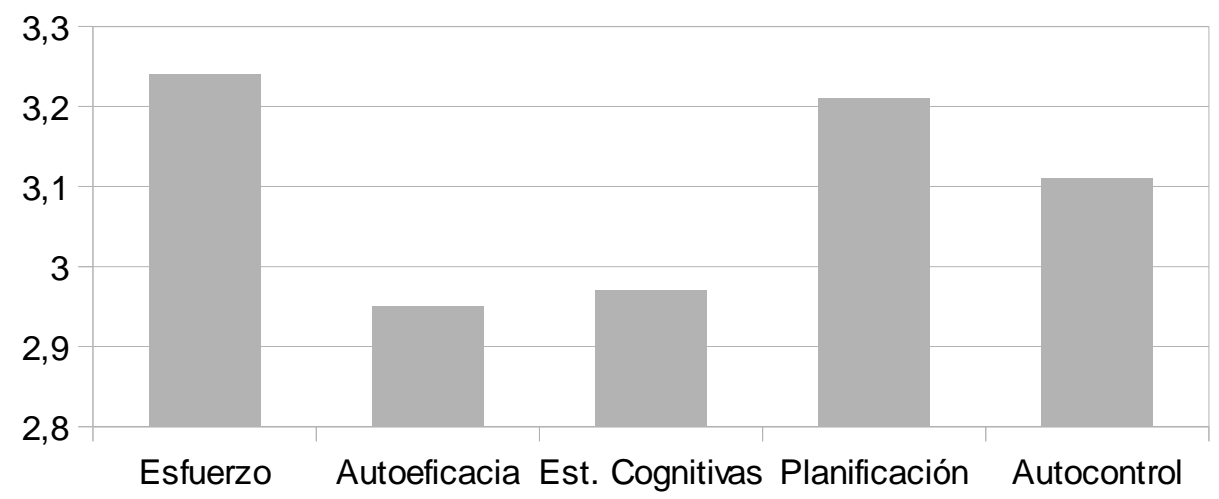

Figura 1. Puntuaciones promedio de los estudiantes en cada uno de los cinco factores del test de O'Neil y Schacter.

Como puede verse en la Figura 1 los dos factores motivacionales reciben la màxima y mínima puntuación (esfuerzo y autoeficacia, respectivamente). De los factores estratégicos, destaca por su mayor puntuación la planificación (estrategia metacognitiva). La Figura 2 pone de manifiesto la mayor dificultad del problema 2 frente al problema 1, totalmente ajustada a las características de mayor complejidad estructural del problema 2.

El cálculo de los coeficientes de correlación producto-momento de Pearson entre los distintos factores del test pone de manifiesto la previsible asociación entre los factores que miden estrategias cognitivas y metacognitivas. Así, se obtienen correlaciones significativas entre: planificación y autocontrol ( $r=.67, p<.001)$, estrategias cognitivas y planificación $(r=.594, p<.001)$, y estrategias cognitivas y autocontrol $(r=.309, p<.05)$. Además aparece también la correlación significativa entre los dos factores que miden la motivación, esto es, autoeficacia y esfuerzo $(r=.671, p<.001)$. 
Con el fin de estudiar si la puntuación obtenida en los problemas puede ser predicha a partir de los cinco factores del test que miden estrategias y motivación en la resolución de problemas, se efectuó un análisis de regresión múltiple tomando como variable dependiente la puntuación de los problemas (problema 1 o problema 2) y como variables independientes (o predictores) cada uno de los cinco factores del test. Para poder diferenciar la contribución de cada factor del test en la puntuación de cada problema, el análisis de regresión se realizó paso a paso (stepwise). En este tipo de regresión los predictores (variables independientes) se introducen uno a uno en orden de importancia, y en cada paso se introduce un nuevo predictor. En la Tabla 1 se muestra este análisis para el problema 1.

Como puede verse la regresión es estadísticamente significativa hasta el tercer paso $(\mathrm{p}<.05)$, esto es, incluyendo los factores planificación (estrategia metacognitiva), esfuerzo y autoeficacia (factores de motivación). Son, por tanto, esos tres factores los que pueden predecir mejor el resultado obtenido en la resolución del problema 1.

Tabla 1. Resultados del análisis de regresión stepwise del problema 1

( $\mathrm{R}^{2}$ representa el cuadrado del coeficiente de correlación múltiple)

\begin{tabular}{cllcc}
\hline $\begin{array}{r}\text { Número } \\
\text { del paso }\end{array}$ & $\begin{array}{c}\text { Variable de } \\
\text { entrada }\end{array}$ & $\mathrm{R}^{2}$ & Prueba F & $\begin{array}{c}\text { Nivel de } \\
\text { significación }(p)\end{array}$ \\
\hline 1 & Planificación & .09 & 6.67 & .01 \\
2 & Esfuerzo & .12 & 4.34 & .02 \\
3 & Autoeficacia & .13 & 3.08 & .03 \\
4 & E.Cognitivas & .14 & 2.45 & .06 \\
5 & Autocontrol & .14 & 1.93 & .10 \\
\hline
\end{tabular}

Según la Tabla 1 la variable predictora más relacionada con la variable dependiente es la planificación, seguida del esfuerzo y la autoeficacia. El cuadrado del coeficiente de correlación múltiple entre la variable criterio (puntuación del problema 1) y las tres variables predictoras (planificación, esfuerzo y autoeficacia) representa la proporción de varianza de la variable dependiente explicada por esas tres variables predictoras. Este valor es de .13 con un nivel de significación $\mathrm{p}<.05$. Ello nos indica que las tres variables combinadas dan cuenta del 13\% de la varianza de la puntuación del problema 1. El 87\% restante debe ser explicado por otras variables no contempladas en este experimento y por la varianza del error. Por otra parte, se observa que R2 pasa de .09, cuando se introduce el factor planificación, a .12, cuando entran en la ecuación planificación y esfuerzo. Finalmente, la adición del factor autoeficacia conduce a un incremento de .01 en el valor global de R2.

En la Tabla 2 se nos muestran los coeficientes de regresión de las tres variables independientes en la ecuación de regresión del problema 1, con su error típico.

Con los valores que figuran en la Tabla 4 puede establecerse que la ecuación que relaciona los factores planificación (P), esfuerzo (E) y autoeficacia (A) con la puntuación en el problema 1 (P1) es 
Tabla 2. Coeficientes de regresión de las tres variables independientes en la ecuación de regresión del problema 1, con su error típico.

\begin{tabular}{ccc}
\hline Variables & Coeficiente de regresión & Error típico \\
\hline Intersección & 1.33 & - \\
Planificación & 3.58 & 1.18 \\
Esfuerzo & -1.00 & .82 \\
Autoeficacia & -.77 & 1.00 \\
\hline
\end{tabular}

$$
P_{1}=1.33+3.58 P-1.00 E-0.77 \mathrm{~A}
$$

Nótese que los coeficientes de regresión de los factores esfuerzo y autoeficacia son negativos. La Tabla 3 presenta los resultados del análisis de regresión stepwise en el caso del problema 2.

Tabla 3. Resultados del análisis de regresión stepwise del problema 2.

\begin{tabular}{ccccc}
\hline $\begin{array}{c}\text { Número } \\
\text { del Paso }\end{array}$ & Variable de entrada & $\mathrm{R}^{2}$ & Prueba F & $\begin{array}{c}\text { Nivel de } \\
\text { significación }(p)\end{array}$ \\
\hline 1 & Esfuerzo & .09 & 6.61 & .01 \\
2 & Autoeficacia & .12 & 4.49 & .02 \\
3 & E.Cognitivas & .13 & 3.20 & .03 \\
4 & Autocontrol & .13 & 2.37 & .06 \\
5 & Planificación & .14 & 1.86 & .11 \\
\hline
\end{tabular}

Puede observarse en la tabla anterior que la regresión es estadísticamente significativa hasta el tercer paso, esto es, incluyendo los factores esfuerzo y autoeficacia (factores de motivación), y estrategias cognitivas. Por consiguiente, esos tres factores son los que pueden predecir mejor el resultado obtenido en la puntuación del problema 2.

De acuerdo con la Tabla 3 la variable predictora más relacionada con la variable dependiente es el esfuerzo, seguida de la autoeficacia y las estrategias cognitivas. Como ya hemos dicho, el cuadrado del coeficiente de correlación múltiple entre la variable criterio (puntuación del problema 2) $\mathrm{y}$ las tres variables predictoras (esfuerzo, autoeficacia y estrategias cognitivas) representa la proporción de varianza de la variable dependiente explicada por esas tres variables predictoras. Este valor es de .13 con un nivel de significación $\mathrm{p}<.05$. Ello nos indica que las tres variables combinadas dan cuenta del $13 \%$ de la varianza de la puntuación del problema 2. El $87 \%$ restante debe ser explicado por otras variables no contempladas en este experimento y por la varianza del error. Por otra parte, se observa que R2 pasa de .09, cuando se introduce el factor esfuerzo, a .12, cuando entran en la ecuación esfuerzo y autoeficacia. Finalmente, la adición del factor estrategias cognitivas conduce a un incremento de .01 en el valor global de R2. La Tabla 4 nos muestra los coeficientes de regresión de las tres variables independientes en la ecuación de regresión del problema 2, con su error típico y su significación estadística.

Tabla 4. Coeficientes de regresión de las tres variables independientes en la ecuación de regresión, con su error típico.

\begin{tabular}{lcc}
\hline \multicolumn{1}{c}{ Variables } & Coeficiente de regresión & Error típico \\
\hline Intersección & 6.60 & - \\
Esfuerzo & -2.92 & 0.98 \\
Autoeficacia & 1.41 & 1.30 \\
E.Cognitivas & 1.38 & 1.67 \\
\hline
\end{tabular}


Con los valores que figuran en la Tabla 4 se puede establecer que la ecuación que relaciona los factores esfuerzo (E), autoeficacia (A) y estrategias cognitivas (EC) con la puntuación en el problema 2 (P2) es

$$
P_{2}=6.60-2.92 E+1.41 A+1.38 E C
$$

Adviértase que el coeficiente de regresión del factor esfuerzo es negativo.

\section{4.- CONCLUSIONES}

Como comentario de carácter introductorio y global, y de acuerdo con la Figura 1 que muestra los resultados del test de estrategias y motivación, los maestros y las maestras en formación dicen esforzarse cuando realizan tareas de resolución de problemas (el factor de mayor puntuación promedio es el esfuerzo), pero tienen conciencia de que su eficacia en dicha tarea es reducida (el factor de menor puntuación promedio es la autoeficacia). Por otra parte, se destacan también en dicha Figura 1 las puntuaciones más elevadas en los factores que evalúan estrategias metacognitivas (planificación y autocontrol) que en el factor estrategias cognitivas. Por tanto, parece que a las primeras se les otorga más importancia en resolución de problemas que a las segundas.

En relación a los factores estratégicos y, a la luz de los resultados de los análisis de regresión múltiple (Tablas 1, 2, 3 y 4), puede decirse para el estudiantado participante en nuestro estudio que: a) El factor planificación (estrategia metacognitiva) resulta ser el factor estratégico más importante para predecir la puntuación de problemas de estructura sencilla (problema 1); b) En la resolución de problemas de estructura más complicada (problema 2), el factor estrategias cognitivas es más relevante que cualquiera de los otros dos que miden estrategias metacognitivas (planificación y autocontrol), aunque es el factor de menor peso entre los que predicen significativamente la puntuación de dicho problema.

Se nos presentan los factores motivacionales de manera sorprendente y contradictoria a primera vista. En los dos problemas, dichos factores contribuyen decisivamente a predecir la puntuación en la resolución. En el problema 1, los dos factores de la motivación (esfuerzo y autoeficacia) tienen coeficientes de regresión negativos. En el problema 2, uno de los dos factores (esfuerzo) tiene coeficiente de regresión negativo, en tanto que el otro (autoeficacia) lo tiene positivo. Una indagación ulterior en los cuadernillos de los estudiantes, en los que se le pedía información adicional relativa a los estudios previos realizados antes de llegar a la facultad, permite concluir que los estudiantes y las estudiantes que han cursado matemáticas en bachillerato, y física y química en la ESO, tienen puntuaciones bajas en el factor esfuerzo y puntuaciones altas en la resolución de problemas. Esto explicaría el coeficiente de regresión negativo: a mayor puntuación en el factor esfuerzo, menor puntuación en resolución de problemas. Una posible interpretación de que el factor autoeficacia tenga coeficiente de regresión negativo en el problema 1, puede ser que un buen número de estudiantes, que tienen conciencia de su escaso éxito en resolución de problemas, hayan obtenido una puntuación alta en dicho problema.

De todo lo dicho hasta aquí se puede concluir que el éxito en la resolución de problemas del profesorado de primaria en formación depende fundamentalmente de dos factores estratégicos, las estrategias cognitivas y la planificación (estrategia metacognitiva), y de dos factores motivacionales, el esfuerzo y la autoeficacia. La contribución de los factores estratégicos es siempre positiva: a mayor uso declarado de estrategias cognitivas y de planificación, mayor puntuación en la resolución de problemas. En cambio, la contribución de un factor motivacional, el esfuerzo, es siempre negativo: a mayor esfuerzo declarado, menor puntuación en resolución de problemas. El otro factor motivacional, la autoeficacia, manifiesta una contribución cambiante, en un tipo de problemas es negativa y en otro tipo es positiva.

No se puede dejar de señalar que, en los análisis de regresión múltiple de los problemas, las tres variables predictoras estadísticamente significativas sólo dan cuenta del $13 \%$ de la varianza de la puntuación de la resolución de los problemas, porcentaje bajo si lo comparamos con otros estudios (Solaz-Portolés y Sanjosé, 2006), lo que pone de manifiesto que las tres variables no son buenas 
predictoras de la puntuación en la resolución de los problemas. Tienen más peso en la explicación de la varianza otros factores no considerados en este estudio y la varianza del error.

Finalmente, se tiene que indicar que la principal limitación de la presente investigación puede derivarse de la naturaleza y el tamaño de la muestra. Los resultados y conclusiones que se obtienen sólo pueden ser válidos stricto sensu para el profesorado de primaria en formación que ha intervenido en ella. Otras limitaciones pueden tener su origen en los instrumentos que se han empleado y, naturalmente, en aquellas variables que no se han podido o sabido controlar y que pueden haber influido en los resultados.

\section{5.- REFERENCIAS}

Beal, C. R. y Stevens, R. H. (2007). Student motivation and performance in scientific problem solving simulations. In R. Luckin, K. R. Koedinger, \& J Greer (Eds.), Artificial intelligence in education: Building technology rich learning contexts that works (pp. 539-541). Amsterdam: IOS Press.

Bielaczyc, K., Pirolli, P. L., y Brown, A. L. (1995). Training in self-explanation and self-regulation strategies: Investigating the effects of knowledge acquisition activities on problem solving. Cognition and Instruction, 13, 221-252.

Brooks, D. W. y Shell, D. F. (2006). Working memory, motivation, and teacher-initiated learning. Journal of Science Education and Technology, 15, 17-30.

Brown, T., McNamara, O., Hanley, U., \& Jones, L. (1999). Primary student teachers' understanding of mathematics and its teaching. British Educational Research Journal, 25, 299-322.

Cacioppo, J. T. y Petty, R. E. (1982). The need of cognition. Journal of Personality and Social Psychology, 42, $116-131$

Conford, I. R. (2002). Learning-to-learn strategies as a basis for effective lifelong learning. International Journal of Lifelong Education, 21, 127-140.

Correa, M. E., Castro, F. y Lira, H. (2004). Estudio descriptivo de las estrategias cognitivas y metacognitivas de alumnos y alumnas de primer año de Pedagogía de la universidad de Bío-Bío. Theoría, 13, 103-110.

Coutinho, A. S. (2006). The Relationship between the Need for Cognition, Metacognition, and Intellectual Task Performance. Educational Research and Reviews, 1 (5), 162-164. Retrieved December 7, 2007, from http://www.academicjournals.org/err/PDF/Pdf2006/Aug/Coutinho.pdf

Coutinho, A. S. (2007). The relationship between goals, metacognition and academic success. Educate, 7, 39-47.

Coutinho, A. S., Wiemer-Hastings, K., Skowronski, J. J., y Britt, M. A. (2005). Metacognition, need for cognition and use of explanations during ongoing learning and problem solving. Learning and Individual Differences, 15, 321-337.

Fergurson-Hessler, M. G. M. y de Jong, T. (1990). Studying physics texts: differences in study processes between good and poor performers. Cognition and Instruction, 7, 41-54.

Flavell, J. H. (1996). El desarrollo cognitivo. Madrid: Prentice Hall.

Hegarty-Harzel, E. y Prosser, M. (1991). Relationship between students' conceptual knowledge and study strategies- part 1: students learning in physics. International Journal of Science Education, 13, 303-312.

Huitt, W. (2001). Why study educational psychology? Educational Psychology Interactive. Valdosta, GA: Valdosta State University. Retrieved May 2008, from http://chiron.valdosta.edu/whuitt/col/intro/whyedpsy.html

Johnstone, A.H., Hogg, W.R., y Ziane, M. (1993). A working memory applied to physics problem solving. International Journal of Science Education, 15, 663-672.

Locke, E. A. y Latham, G. P. (1990). A theory of goal setting and task performance. Englewood Cliffs, NJ: Prentice Hall.

Marcou, A., y Philippou, G. (2005). Motivational beliefs, self-regulated learning and mathematical problem solving. In H. L. Chick \& J. L. Vincent (Eds.), Proceedings of the 29 Conference of the International Group for the Psychology of Mathematics Education (pp. 297-304). Melbourne: PME.

Neto, A. J. y Valente, M. (1997). Problem solving in physics: Towards a metacognitively developed approach. Paper presented at the Annual Meeting of the National Association for Research in Science Teaching, Oak Brook, IL. 
O’Neil, H.F., Jr, \& Schacter, J. (1997). Test Specifications for Problem - Solving Assessment (CSE Tech. Rep. $\mathrm{N}^{\circ}$ 463). Los Angeles: University of California, CRESST.

Schraw, G., Crippen, K. J. y Hartley, K. D. (2006). Promoting self-regulation in science education: Metacognition as part of a broader perspective on learning. Research in Science Education, 36(1-2), 111-139.

Schraw, G., Brooks, D. W. y Crippen, K. J. (2005). Improving chemistry teaching using an interactive compensatory model of learning. Journal of Chemical Education, 82(4), 637-640.

Simon, M. (1993). Prospective elementary teachers' knowledge of division. Journal for Research in Mathematics Education ,24, 233-254.

Solaz-Portolés, J. J. y Sanjosé, V. (2006). ¿Podemos predecir el rendimiento de nuestros alumnos en resolución de problemas? Revista de Educación, 339, 693-710.

Solaz-Portolés, J. J., Sanjosé, V., \& Gangoso, Z. (2013). La investigación en resolución de problemas instruccionales. Efectos de variables del problema y de las variables cognitivas, metacognitivas y motivacionales del resolutor. En J. Benegas, M.C. Pérez de Landazábal y J. Otero (Eds.), El aprendizaje activo de la Física Básica Universitaria (pp. 95-118). Santiago de Compostela: Andavira Editora.

Song, H. D. y Grabowski, B. L. (2006). Stimulating intrinsic motivation for problem solving using goal oriented contexts and peer composition. Educational Technology Research \& Development, 54, 445-466.

Taplin, M. (1998). Preservice teacher's problem-solving processes. Mathematics Education Research Journal, $10,59-76$.

Teong, S. K. (2003). The effect of metacognitive training on mathematical word-problem solving. Journal of Computer Assisted Learning, 19, 46-55.

Valverde, A. G., \& Castro, E. (2009). Actuaciones de maestros en formación en la resolución de problemas de proporcionalidad directa. En M.J. González, M.T. González \& J. Murillo (Eds.), Investigación en Educación Matemática XIII (pp. 523-531). Santander: SEIEM.

Weinstein, C. E. y Meyer, D. K. (1994). Learning strategies, teaching and testing. The International Encyclopedia of Education, $2^{\text {nd }}$ Edition (pp. 3335- 3340. Oxford: Pergamon Press.

Wieth, M., y Burns, B. D. (1999). Motivation in insight versus incremental problem solving. In L. R. Gleitman \& A. K. Joshi (Eds.), Proceedings of the 22 Annual Conference of the Cognitive Science Society (pp. 559-564). Mahwah, N. J.: L. Erlbaum.

Wolters, C. A. y Rosenthal, H. (2000). The relation between students' motivational beliefs and their use of motivational regulation strategies. International Journal of Educational Research, 33, 801-820. 